\title{
Electronic and chemical properties of cathode structures using 4,7-diphenyl-1,10-phenanthroline doped with rubidium carbonate as electron injection layers
}

\author{
Mei-Hsin Chen, ${ }^{1}$ Yu-Hung Chen, ${ }^{1}$ Chang-Tin Lin, ${ }^{1}$ Guan-Ru Lee, ${ }^{1}$ Chih-I Wu, ${ }^{1, a)}$ \\ Dong-Seok Leem, ${ }^{2}$ Jang-Joo Kim, ${ }^{2}$ and Tun-Wen $\mathrm{Pi}^{3}$ \\ ${ }^{1}$ Department of Electrical Engineering and Graduate Institute of Photonics and Optoelectronics, \\ National Taiwan University, Taipei, Taiwan 10617, Republic of China \\ ${ }^{2}$ Department of Materials Science and Engineering, Center for Organic Light Emitting Diode, \\ Seoul National University, Seoul 151-742, Republic of Korea \\ ${ }^{3}$ National Synchrotron Radiation Research Center, Hsinchu, Taiwan 300, Republic of China
}

(Received 9 April 2009; accepted 5 May 2009; published online 11 June 2009)

\begin{abstract}
The electronic properties and chemical interactions of cathode structures using 4,7-diphenyl-1, 10-phenanthroline (Bphen) doped with rubidium carbonate $\left(\mathrm{Rb}_{2} \mathrm{CO}_{3}\right)$ as electron injection layers were investigated. Current-voltage characteristics reveal that the devices with Bphen $/ \mathrm{Rb}_{2} \mathrm{CO}_{3} / \mathrm{Al}$ as cathode structures possess better electron injection efficiency than those with cathode structures of $\mathrm{Bphen} / \mathrm{LiF} / \mathrm{Al}$. Ultraviolet and $\mathrm{x}$-ray photoemission spectroscopy shows that $n$-type doping effects resulting from $\mathrm{Rb}_{2} \mathrm{CO}_{3}$ and the gap states created by aluminum deposition are both keys to the improved carrier injection efficiency. Moreover, theoretical calculation indicates that the chemical reaction between aluminum and the nitrogen atoms in Bphen is the origin of the gap states. (C) 2009 American Institute of Physics. [DOI: 10.1063/1.3143718]
\end{abstract}

\section{INTRODUCTION}

Steady advances have been made in organic lightemitting diode (OLED) since the pioneering work of Tang and Van Slyke. ${ }^{1}$ Tris(8-hydroxyquinoline) aluminum $\left(\mathrm{Alq}_{3}\right)$, which possesses superior film stability and thermal endurance, is one of the most reliable hosts for achieving different colors and enhancing luminous efficiency. Although $\mathrm{Alq}_{3}$ is one of the most reliable hosts in achieving high efficiency in OLEDs, its low electron mobility of $5.5 \times 10^{-6} \mathrm{~cm}^{2} \mathrm{v}^{-1} \mathrm{~s}^{-1}$ and intrinsic degradation ${ }^{2-4}$ are responsible for the undesirable high resistance appearing in $\mathrm{Alq}_{3}$-based devices. Thus, 4,7-diphenyl-1,10-phenanthroline (Bphen) is one of the alternatives to be used as an electron transport layer (ETL) in OLEDs to enhance their performance based on its higher electron mobility of $5 \times 10^{-4} \mathrm{~cm}^{2} \mathrm{v}^{-1} \mathrm{~s}^{-1}$. ${ }^{5-9}$ The ionization energy of Bphen is $6.4 \mathrm{eV}$. The deeper highest occupied molecular orbital (HOMO) level implies that the material is stable against oxidization as well as can be used as a holeblocking layer in OLEDs and consequently increase the efficiency of the devices.

In addition, an effective cathode and electron injection layer (EIL) to inject the electrons to ETL is another key issue to provide efficient electron currents in the devices. Conductive doping, which can result in reducing the electron injection barrier effectively and therefore improve device performance, plays an important role in making OLEDs. It is well known that the work function of the cathode can be reduced significantly by inserting alkali metals, even with a very thin layer, ${ }^{10}$ between organic layers and cathode. Several studies

\footnotetext{
${ }^{\text {a) }}$ Author to whom correspondence should be addressed. Tel.: 886-233663656. FAX: 886-2-23677467. Electronic mail: chihiwu@cc.ee.ntu.edu.tw.
}

have been focused on the physics and chemistry at interfaces between cathode materials and $\mathrm{Alq}_{3} .{ }^{11-14}$ However, little research is reported on the electronic and chemical properties of Bphen, which process better electrical properties than that of $\mathrm{Alq}_{3}$, at the cathode interfaces. In this paper, we report the mechanisms of carrier injection in Bphen doped with a newly developed rubidium carbonate $\left(\mathrm{Rb}_{2} \mathrm{CO}_{3}\right) .{ }^{15}$ By means of $J-V$ characteristics of devices and ultraviolet and $\mathrm{x}$-ray photoemission spectroscopy (UPS and XPS), the electronic structures and the interfacial chemical reaction are investigated.

\section{EXPERIMENTAL AND THEORETICAL}

The devices were fabricated on indium-tin-oxide (ITO)coated glass substrates as anodes. $N, N^{\prime}$-bis(1-naphthyl)$N, N^{\prime}$-diphenyl-1, $1^{\prime}$-biphenyl-4, $4^{\prime}$-diamine (NPB) and Bphen were used as hole transport layers and ETLs, respectively. The thicknesses of NPB and Bphen are $80 \mathrm{~nm}$ in each layer. ITO substrates were cleaned by acetone, methanol, and UV ozone before loaded into the deposition chamber. The cathode metal was thermally evaporated on top of the organic film through a shadow mask to define an active area of $1 \mathrm{~mm}^{2}$. The current density-voltage $(J-V)$ characteristics of the devices were measured by a Keithley 2636 source-meter unit, which has high sensitivity at low current. All the devices were measured right after fabrication.

Photoemission experiments were carried out in a three interconnected ultrahigh vacuum chambers, two for deposition and the other for spectroscopy analysis. The Fermi level of the system was measured on a gold film cleaned by very low dose of Ar sputter. All organic materials were deposited through thermal evaporation in the deposition chamber and were then transferred in situ to the analysis chamber. The 


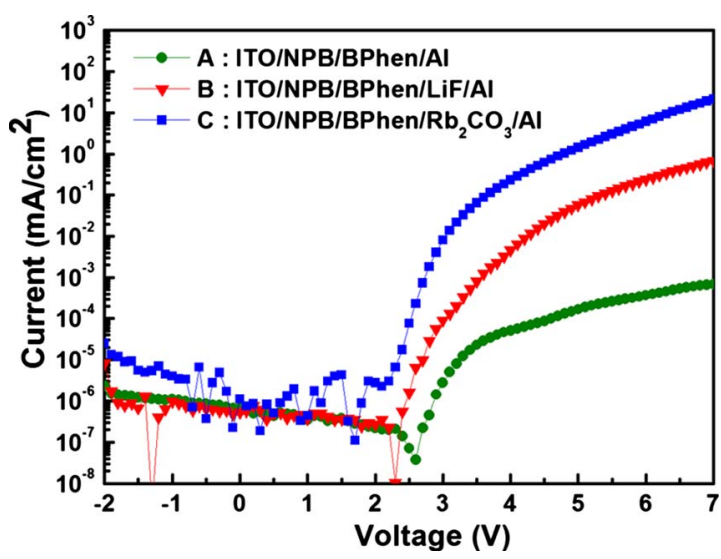

FIG. 1. (Color online) The $J-V$ characteristics of the devices.

valence band UPS were measured via $\mathrm{He} \mathrm{I}(21.2 \mathrm{eV})$ and $\mathrm{He}$ II $(40.8 \mathrm{eV})$ as excitation sources at a base pressure at $10^{-10}$ Torr. The energy position changes of the HOMO with the doping process could be observed through aligning the Fermi level. The changes in the vacuum level could be observed with the sample biased at $-6 \mathrm{~V}$ to observe the onset at lower binding energy in UPS. To get a better resolution, XPS were performed at National Synchrotron Radiation Research Center (NSRRC), Hsinchu, Taiwan, R.O.C. The highbrightness photon beam is provided by the U5-SGM monochromator with photon energy in the range of $60-1000 \mathrm{eV}$. The core level spectra were measured with the photon energies chosen to optimize the signal-to-noise ratio. The energy spectra of photoelectrons were collected with a $125 \mathrm{~mm}$ hemispherical analyzer with overall resolution better than 0.1 $\mathrm{eV}$. By comparing the changes of binding energy in core level photoemission spectra, chemical reactions at the interfaces during the deposition process could be understood.

To analyze origins of the electronic states in organic materials, quantum-chemical calculation package, GAUSSIAN03, was used to compute the molecular orbital. ${ }^{16,17}$ The structures of the molecules were optimized using Hartree-Fock method with 6-31G basis set and the molecular orbitals were calculated with B3LYP exchange correlation function. The theoretical density of states or simulated UPS spectra were then calculated by convoluting the molecular energy levels with Gaussian function using the full width at half maximum of $0.5 \mathrm{eV}$, which represents the broadening of discrete molecular levels due to the amorphous nature of organic films, vibronic origins, and instrumental broadening. ${ }^{18,19}$

\section{RESULTS AND DISCUSSION}

Figure 1 shows the performance of devices with current versus voltage curves in log scale. The effects of $\mathrm{Rb}_{2} \mathrm{CO}_{3}$, increasing electron injection ability from metal cathodes into Bphen, were investigated via a series of devices: (1) ITO/ $\mathrm{NPB}(80 \mathrm{~nm}) / \mathrm{Bphen}(80 \mathrm{~nm}) / \mathrm{Al}$ (device A); (2) ITO/NPB(80 $\mathrm{nm}) / \mathrm{Bphen}(80 \mathrm{~nm}) / \mathrm{LiF}(1 \mathrm{~nm}) / \mathrm{Al}$ (device B); and (3) ITO/ $\mathrm{NPB}(80 \mathrm{~nm}) / \mathrm{Bphen}(80 \mathrm{~nm}) / \mathrm{Rb}_{2} \mathrm{CO}_{3}(1 \mathrm{~nm}) / \mathrm{Al}$ (device C). Device A, with aluminum only as a cathode, is expected to have large electron injection barrier height and shows poor characteristics. Device B, consisting of $\mathrm{LiF}$ with aluminum, a commonly used effective cathode structure, was fabricated

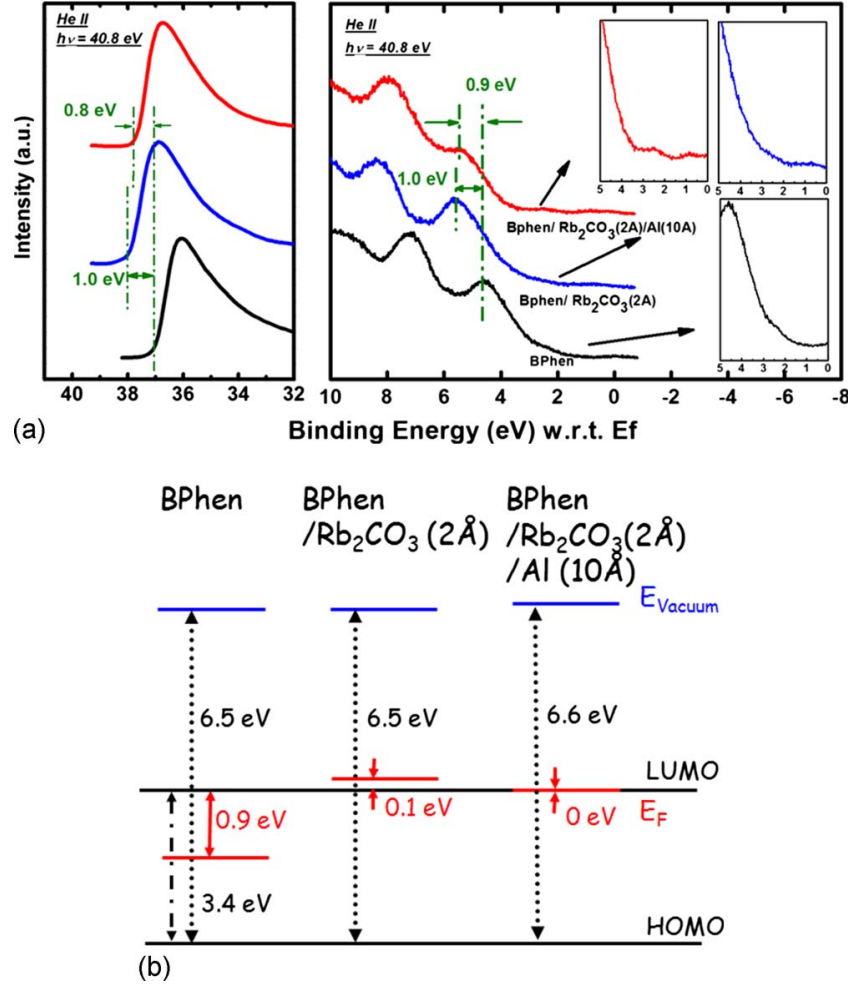

FIG. 2. (Color online) (a) UPS spectra, near the onset and valence bands, of Bphen with $\mathrm{Rb}_{2} \mathrm{CO}_{3}$ and aluminum deposition and (b) the associated energy band diagram.

for comparison. Device $\mathrm{C}$ was inserted a thin layer of $\mathrm{Rb}_{2} \mathrm{CO}_{3}$ as an EIL on top of Bphen, which drastically improves the device performance. The current increases by almost two orders of magnitude as compared to those with the commonly used $\mathrm{LiF} / \mathrm{Al}$ cathode structure. The effect of $\mathrm{Rb}_{2} \mathrm{CO}_{3}$ doped Bphen on the device performance was further investigated with UPS and XPS.

Figure 2(a) reveals the UPS spectra near the onset as well as the HOMO level of Bphen films with subsequent deposition of ultrathin layers of $\mathrm{Rb}_{2} \mathrm{CO}_{3}$ and aluminum. Energy band diagrams deduced from UPS are shown in Fig. 2(b). The thickness of the deposition is obtained from the quartz oscillator calibrated with density and the geometric factor. For pristine Bphen, the edge of the HOMO is about $2.5 \mathrm{eV}$ below the Fermi level and the ionization energy is 6.5 $\mathrm{eV}$, which is consistent with the reported data. ${ }^{20}$ With $0.2 \mathrm{~nm}$ of $\mathrm{Rb}_{2} \mathrm{CO}_{3}$ deposited on Bphen, the valence-band features of Bphen are still clearly seen in the UPS spectrum, while the HOMO and other peaks rigidly shift toward lower binding energies by $1.0 \mathrm{eV}$. The result indicates that the Fermi level of Bphen after doped with $\mathrm{Rb}_{2} \mathrm{CO}_{3}$ shifts toward the lowest unoccupied molecule orbital (LUMO), showing $\mathrm{Rb}_{2} \mathrm{CO}_{3}$ as $n$-type dopants in Bphen films. The onset of the UPS spectra, representing the vacuum level of the films, also moves toward lower binding energy by $1.0 \mathrm{eV}$. The electron injection barrier, the energy level between the Fermi level to the LUMO of organic materials, is therefore greatly reduced. This is different from the case of $\mathrm{LiF} / \mathrm{Al}$ cathode structures in which the injection barrier lowering only happens after aluminum deposition. The deposition of aluminum pulls down the Fermi of the sample a little bit due to the higher work 


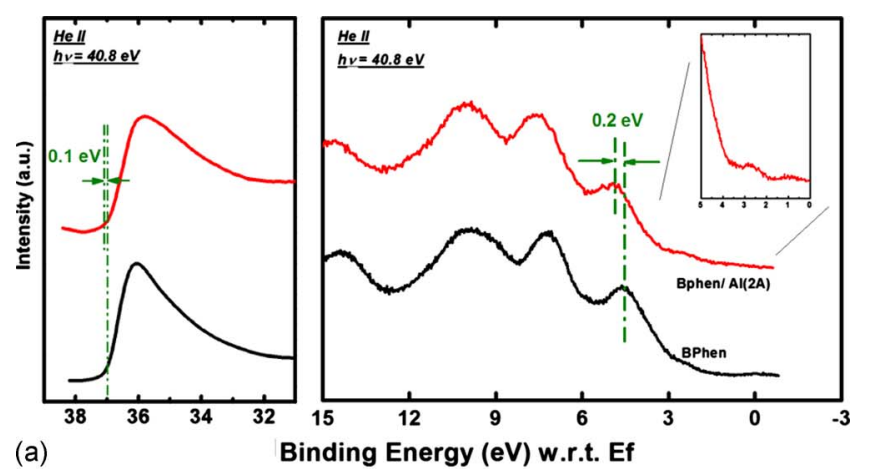

(a)

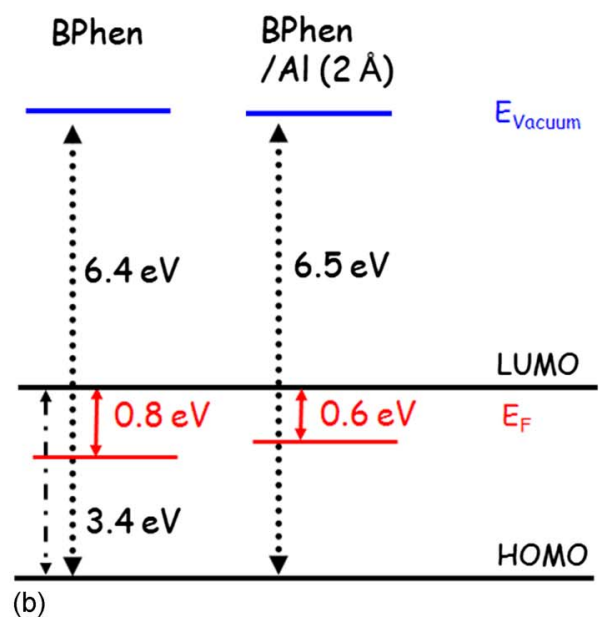

FIG. 3. (Color online) (a) UPS spectra, near the onset as well as valence bands, of pristine Bphen with deposition of aluminum and (b) the associated energy band diagram.

function of aluminum $(4.2 \mathrm{eV})$ as compared to the electron affinity of Bphen $(3.0 \mathrm{eV})$. Given that the band gap of Bphen is $3.4 \mathrm{eV}$, the final position of the Fermi level at the interface close to the cathode is at the vicinity of the LUMO of Bphen. Furthermore, as shown in the inset of Fig. 2(a), with aluminum deposition on Bphen $/ \mathrm{Rb}_{2} \mathrm{CO}_{3}$ there are two features in the gap appearing within $3 \mathrm{eV}$ below the Fermi level. These gap states were not observed in the UPS spectra of pristine Bphen. The origins of these gap states were then further investigated.

Figures 3(a) and 3(b) show the valence-band spectra of aluminum deposited directly on pristine Bphen and associated energy band diagrams, respectively. The gap states that appear in Fig. 2(a) can also be observed with the deposition of aluminum on pristine Bphen. The result demonstrates that these gap states are generated by the interaction between Bphen and aluminum, without the participation of $\mathrm{Rb}_{2} \mathrm{CO}_{3}$. However, the Fermi level moves only slightly closer to the LUMO by $0.2 \mathrm{eV}$ and still about $0.6 \mathrm{eV}$ below the LUMO, resulting in poor electron injection property of device $\mathrm{A}$ as shown in Fig. 1. Although gap states can be formed to provide extra states for electron in this case, device performance is also dominated by the electron injection barrier. The role of $\mathrm{Rb}_{2} \mathrm{CO}_{3}$ in the device is to move the Fermi level close to the LUMO of Bphen. Combining the results from $J-V$ characteristics and UPS spectra, the improved performance of device $\mathrm{C}$ with $\mathrm{Rb}_{2} \mathrm{CO}_{3}$ and $\mathrm{Al}$ in cathode structure indicates that the efficiency of electron injection may result from both

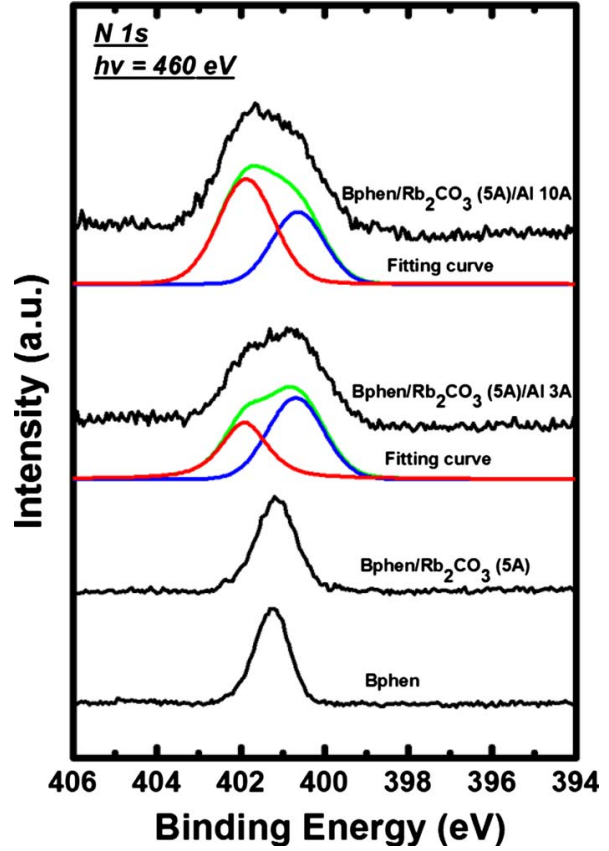

FIG. 4. (Color online) XPS spectra of the nitrogen $1 s$ core-level spectra of pristine Bphen with incremental depositions of $\mathrm{Rb}_{2} \mathrm{CO}_{3}$ and aluminum.

lowering the carrier injection barriers and existence of gap states. In this study, other metals (such as silver, magnesium, and gold) were also tested as cathode materials with Bphen and gap states were formed only with aluminum. More investigations need to be carried out to discover the general relationship between gap states and carrier injection in OLEDs.

The interfacial chemistry was studied by XPS. The incremental depositions of aluminum were carried out so that the interfacial reaction could be investigated via photoemission spectroscopy. The XPS spectra were normalized to constant intensity for comparison purposes. With deposition of aluminum, the rubidium $3 d$ core level in XPS spectra did not change significantly, showing that rubidium atoms are not involved in the interaction to form the gap states. Figure 4 shows the nitrogen $1 \mathrm{~s}$ core-level spectra of pristine Bphen, and spectra with incremental depositions of $\mathrm{Rb}_{2} \mathrm{CO}_{3}$ and aluminum. These nitrogen $1 s$ core level spectra were fitted with two Gaussian peaks. The bottom spectra show that the binding energy of the nitrogen atom $1 s$ core level of pristine Bphen was $401.2 \mathrm{eV}$. With an increasing thickness of a few angstroms of $\mathrm{Rb}_{2} \mathrm{CO}_{3}$, the line width of the nitrogen $1 s$ core level was slightly broadened, which is due to the disorder from the deposition of foreign species. No noticeable new feature appeared in nitrogen $1 s$ core level with $\mathrm{Rb}_{2} \mathrm{CO}_{3}$ mixed in Bphen. However, with the incremental deposition of aluminum from 3 to $10 \AA$, the core level of nitrogen $1 s$ reveals significant changes. The nitrogen $1 s$ core level of pristine Bphen shifts slightly toward a lower binding energy and a new component of nitrogen $1 s$ core level appears at a higher binding energy with deposition of aluminum. The intensity of the peak at higher binding energy, about $402 \mathrm{eV}$, increases as the amount of aluminum on the surface increases. The higher binding energy of the new core level feature indicates the loss of electrons around the nitrogen 


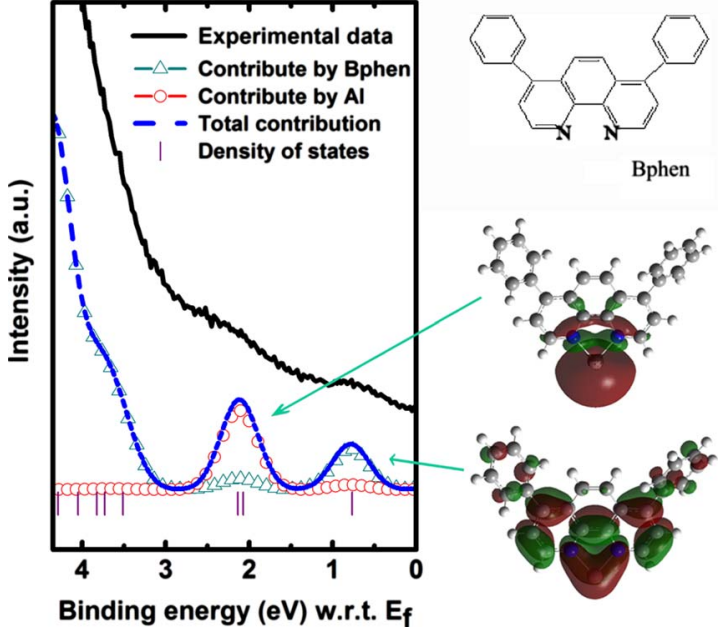

FIG. 5. (Color online) The experimental and simulated UPS spectra. The chemical structure of Bphen and the molecular orbitals associated with the two gap states features are shown on the right.

atom after aluminum deposition. From the molecular structure it is known that nitrogen atoms in Bphen have a lone pair of electrons. Therefore the new feature of nitrogen $1 s$ core level located at a higher binding energy of $402 \mathrm{eV}$ could be attributed to the formation of bonding between nitrogen atoms in Bphen and incoming aluminum atoms. To investigate the correlation between the chemical reaction of aluminum with Bphen and the formation of gap states, we carried out the quantum chemistry calculation on the molecular orbital.

Figure 5 shows the comparison of simulated density of states and the experimental UPS data with an aluminum atom attached to a Bphen molecule. The lowest energy level configuration was attained with the aluminum atom bonded to the nitrogen atoms with lone pairs of electrons. Two gap state features are also obtained from the theoretical calculation and the energy distribution of the gap states agrees well with the experimental data. The spatial distributions of the molecular orbitals associated with these gap states are shown at the right hand side of Fig. 5, with an aluminum atom at the bottom of the Bphen molecules. It is clear that the gap states located at about $2 \mathrm{eV}$ below the Fermi level are mainly contributed by the aluminum atom, whereas the second gap states features at about $1 \mathrm{eV}$ are extended between aluminum and Bphen. No such reaction was found between Bphen and $\mathrm{Rb}$ atoms with similar theoretical calculation, indicating that the gap states are caused by the chemical interaction between the lone pair of electrons in the Bphen and aluminum atoms.

\section{SUMMARY}

In summary, we report the electronic properties and chemical interactions of the cathode structures using Bphen with a thin layer of $\mathrm{Rb}_{2} \mathrm{CO}_{3}$ as EILs. $J$ - $V$ characteristics of the devices indicate that the devices with $\mathrm{Bphen} / \mathrm{Rb}_{2} \mathrm{CO}_{3} / \mathrm{Al}$ as cathode structures possess better electron injection efficiency than those with cathode structure of Bphen/LiF/Al. Photoemission spectroscopy shows that the $n$-type doping effects resulting from $\mathrm{Rb}_{2} \mathrm{CO}_{3}$ and the gap states created by aluminum deposition are the keys to the improved carrier injection efficiency. Theoretical calculation indicates that the chemical reaction of aluminum and the nitrogen atoms in Bphen is the origin of the gap states.

\section{ACKNOWLEDGMENTS}

This work was partially supported by the National Science Council, the Republic of China, under contract no. NSC 95-2745-M-002-011. We are grateful to Computer and Information Networking Center, National Taiwan University for the support of high-performance computing facilities.

${ }^{1}$ C. Tang and S. Van Slyke, Appl. Phys. Lett. 51, 913 (1987).

${ }^{2}$ H. H. Fong, W. C. H. Choy, K. N. Hui, and Y. J. Liang, Appl. Phys. Lett. 88, 113510 (2006).

${ }^{3}$ H. H. Fong, K. C. Lun, and S. K. So, Jpn. J. Appl. Phys., Part 2 41, L1122 (2002).

${ }^{4}$ S. C. Tse, H. H. Fong, and S. K. So, J. Appl. Phys. 94, 2033 (2003).

${ }^{5}$ S. Naka, H. Okada, H. Onnagawa, and T. Tsutsui, Appl. Phys. Lett. 76, 197 (2000).

${ }^{6}$ M. Y. Chan, C. S. Lee, S. L. Lai, M. K. Fung, F. L. Wong, H. Y. Sun, K. M. Lau, and S. T. Lee, J. Appl. Phys. 100, 094506 (2006).

${ }^{7}$ M. A. Khan W. Xu, K.-ul. Haq, Y. Bai, X. Y. Jiang, Z. L. Zhang, and W. Q. Zhu, J. Appl. Phys. 103, 014509 (2008).

${ }^{8}$ S. L. Lai, M. Y. Chan, M. K. Fing, C. S. Lee, and S. T. Lee, Appl. Phys. Lett. 90, 203510 (2007).

${ }^{9}$ K. Walzer, B. Meanning, M. Pfeiffer, and K. Leo, Chem. Rev. 107, 1233 (2007).

${ }^{10}$ A. Neumann, S. L. M. Schrieder, and K. Chrisman, Phys. Rev. B 51, 17007 (1995).

${ }^{11}$ J. Kido and T. Matsumoto, Appl. Phys. Lett. 73, 2866 (1998).

${ }^{12}$ H. Ding and Y. Gao, Appl. Phys. Lett. 86, 213508 (2005).

${ }^{13}$ C. I. Wu, C. T. Lin, Y. H. Chen, M. H. Chen, Y. J. Lu, and C. C. Wu, Appl. Phys. Lett. 88, 152104 (2006).

${ }^{14}$ M. H. Chen and C. I. Wu, J. Appl. Phys. 104, 113713 (2008).

${ }^{15}$ D. S. Leem, S. Y. Kim, J. J. Kim, M. H. Chen, and C. I. Wu, Electrochem. Solid-State Lett. 12, J8 (2009).

${ }^{16}$ M. J. Frisch, G. W. Trucks, H. B. Schlegel et al., Gaussian03, Revision E.01, Gaussian, Inc., Wallingford CT, 2004.

${ }^{17}$ N. M. O'Boyle, A. L. Tenderholt, and K. M. Langner, J. Comput. Chem. 29, 839 (2008).

${ }^{18}$ I. G. Hill, A. Kahn, J. Cornil, D. A. Santos, and J. L. Bredas, Chem. Phys. Lett. 317, 444 (2000).

${ }^{19}$ C. I. Wu, G. R. Lee, C. T. Lin, Y. H. Chen, Y. H. Hung, W. G. Liu, C. C. Wu, K. T. Wong, and T. C. Chao, Appl. Phys. Lett. 87, 242107 (2005).

${ }^{20}$ M. Y. Chan, S. L. Lai, K. M. Lau, C. S. Lee, and S. T. Lee, Appl. Phys. Lett. 89, 163515 (2006). 